\title{
Alignment of energy levels in dye/semiconductor interfaces by $G W$ calculations: Effects due to coadsorption of solvent molecules
}

\author{
Carla Verdi* \\ Dipartimento di Fisica e Astronomia, Università di Padova, via Marzolo 8, I-35131 Padova, Italy \\ Edoardo Mosconi and Filippo De Angelis \\ Istituto CNR di Scienze e Tecnologie Molecolare, via Elce di Sotto 8, I-06123 Perugia, Italy \\ Margherita Marsili \\ Dipartimento di Fisica e Astronomia, Università di Padova, via Marzolo 8, I-35131 Padova, Italy \\ P. Umari \\ Dipartimento di Fisica e Astronomia, Università di Padova, via Marzolo 8, I-35131 Padova, Italy \\ and CNR-IOM Democritos, Theory@Elettra group, Basovizza, Trieste, Italy
}

(Received 16 April 2014; revised manuscript received 17 September 2014; published 6 October 2014)

\begin{abstract}
The performance of dye-sensitized solar cells is tightly linked to the relative energy level alignment of its constituents. In this paper the electronic properties of a model of dye-sensitized solar cell are studied by accurate first-principle calculations taking into account many-body effects beyond density-functional theory. The cell model includes one layer of co-adsorbed solvent (water or acetonitrile) molecules. Solvent molecules induce an upwards energy shift in the $\mathrm{TiO}_{2}$ bands; such a shift is larger in the case of acetonitrile. The accurate determination of the energy levels allows the theoretical estimation of the maximum attainable open circuit voltage $\left(V_{\mathrm{oc}}\right)$.
\end{abstract}

DOI: 10.1103/PhysRevB.90.155410

PACS number(s): 73.20.At

\section{INTRODUCTION}

Dye-sensitized solar cells [1] (DSSCs) are a promising alternative to conventional solar cells, attracting considerable attention and research effort thanks to their low cost in terms of raw material and production processes, in spite of lower conversion efficiencies than in conventional solar cells [2]. In a DSSC light is absorbed by dye molecules adsorbed to the surface of a wide-gap semiconductor, usually $\mathrm{TiO}_{2}$. After light absorption, the photoexcited electrons of the dye are transferred into the conduction band of the semiconductor and then travel to the front electrode. A red-ox couple, usually iodide/triiodide $\left(\mathrm{I}^{-} / \mathrm{I}_{3}^{-}\right)$in an organic solvent, typically acetonitrile, reduces the oxidized dye back to its neutral state and is then regenerated at the counter electrode (usually made by metallic Pt) thus closing the circuit (Fig. 1). The correct alignment of the electronic energy levels within and between each constituent of the device is thus at the very basis of the device functioning.

The main physical parameters of solar cells are the shortcircuit current under illumination $J_{\mathrm{sc}}$ and the open-circuit voltage under illumination $V_{\mathrm{oc}}$. In DSSCs $J_{\mathrm{sc}}$ is determined by the optical gap of the dye molecule and by the conversion efficiency for the produced electron-hole pairs; $V_{\text {oc }}$ is given by the energy difference between the quasi-Fermi level of the conduction band $(\mathrm{CB})$ of the semiconductor and the energy level of the redox couple (see Fig. 1). While the latter is uniquely determined by the chosen redox couple and solvent, the first depends on several factors such as the exposed surface, the dye, and the solvent [3]. Due to the complexity of the

\footnotetext{
*Present address: Department of Materials, University of Oxford, Parks Road, Oxford OX1 3PH, United Kingdom.
}

system, the design of new DSSCs would benefit from the possibility of calculating $V_{\mathrm{oc}}$ for realistic model structures which should include the presence of a solvent. Here, we show how accurate first-principles modeling of DSSCs can give estimates of the maximum achievable $V_{\mathrm{oc}}$ in good agreement with experimental data.

When structural properties are addressed, accurate firstprinciples modeling of DSSCs can be achieved through density functional theory (DFT). However, when electronic or optical excitations are considered, DFT usually yields rather poor results [4]. In contrast, methods based on first-principles many-body perturbation theory (MBPT), although much more computationally demanding, provide electronic and optical properties in very good agreement with experimental data [4]. In particular, the GW approach, in which the self-energy is expressed as the product of the one-body Green's function $\mathrm{G}$ with the screened Coulomb interaction $\mathrm{W}$, gives accurate results for electronic energy levels [5].

Recently, the GW method has been successfully used for investigating $\mathrm{TiO}_{2}$ crystals and slabs, for investigating a crystalline dye structure [6], for modeling the constituents of a DSSC [7], and for evaluating the electronic levels of a model describing an all-organic dye adsorbed on a $\mathrm{TiO}_{2}$ slab [8]. Thus, the next step is to include in a realistic model structure also the solvent, an essential component of such devices. As large simulation cells are needed for this task, we adopt here a GW implementation which is particularly suitable for large systems $[9,10]$. We address the prototypical all-organic dye (4-Diphenylamino)phenylcyanoacrylic acid (usually referred to as L0) [11-13]. Its synthesis is described in Ref. [14] and it led to efficiencies ranging from $1.3 \%$ to $2.7 \%$ depending on cell details as the used electrolyte [14-16].

One experimental description of a DSSC using the L0 dye is given in Ref. [15]. Atop of fluorine-doped tin oxide 


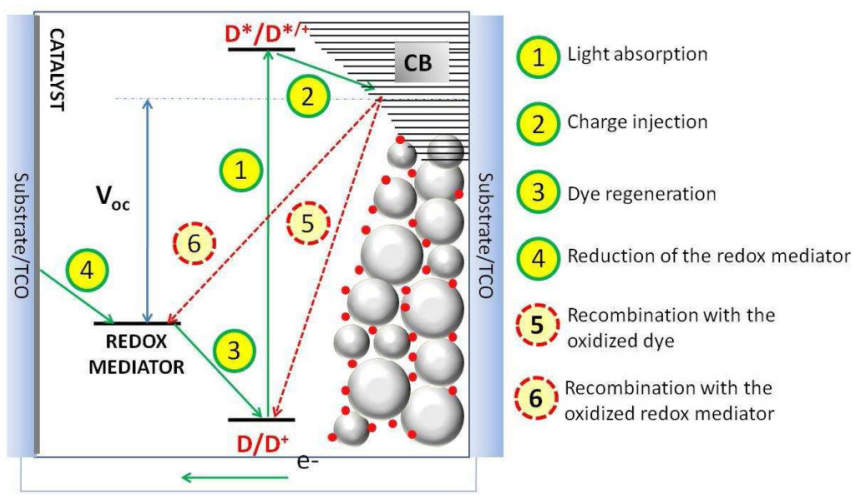

FIG. 1. (Color online) Energy levels scheme and device operation of a DSSC (green-full and red-dashed lines indicate forward and back electron transfer processes respectively). The open-circuit voltage $V_{\text {oc }}$ is also shown.

(FTO), which behaves as a transparent conducting medium, a transparent nanocrystalline film is grown starting from a $\mathrm{TiO}_{2}$ paste composed by $\sim 25 \mathrm{~nm}$ colloidal particles. These particles would exhibit the (101) as the majority surface [17], and this is the surface we investigate. Surface steps have been considered in Ref. [18] finding no enhanced adsorption. After dye sensitization a liquid electrolyte is introduced, and the system is completed by a platinized FTO layer forming the counter electrode. As first-principles simulations, in particular those regarding the evaluation of excitation properties, are typically limited to systems up to a very few hundreds of atoms, we must consider quite a simplified model. Indeed, we focus only on the dye $/ \mathrm{TiO}_{2}$ interface. This is justified by the almost negligible potential drop at the interfaces with the FTO layers. These are of the Schottky's barrier kind and are considered in Ref [19]. Therefore, we use the conduction band minimum of the $\mathrm{TiO}_{2}$ slab for approximating the quasi Fermi level of the transparent conducting medium. Room temperature molecular dynamics simulations, based on DFT and on force fields, of the $\mathrm{TiO}_{2}$ /liquid acetonitrile interface showed that there is a clear arrangement in layers up to $\sim 12 \AA$ above the $\mathrm{TiO}_{2}$ surface, and that the first layer is co-adsorbed occupying all available sites with the acetonitrile molecules presenting the same orientation [20]. Similar results were also found in the case of water [21]. The solvent monolayer does not hamper the dye regeneration process. Indeed, the $\mathrm{I}^{-}$density profiles within $\mathrm{TiO}_{2}$ /liquid acetonitrile interface were studied in Ref. [22]. Depending on the chosen cation, different $\mathrm{I}^{-}$ density profiles were obtained, such profiles showing peaks in the region where the the dye groups involved in the regeneration process are typically located [22].

A consequence of the solvent molecules adsorption on the $\mathrm{TiO}_{2}$ surface is the formation of an aligned dipole layer. This dipole layer, if acting in the proper direction, can favorably shift the semiconductor bands [22-24]. In semisquarylium dye sensitized solar cells no significant band shifts are reported, although the solvent layer (in this case methoxypropionitrile) is important for blocking electron-hole recombination by damping $\mathrm{TiO}_{2}$ surface states [25].

We previously investigated the $\mathrm{L} 0 / \mathrm{TiO}_{2}$ interface without solvent molecules in Ref. [8], and now we explicitly consider the cases of acetonitrile and water solvents, in order to understand how the different degree of polarity of the solvent molecules can affect the energy shifts. In this study we have limited our investigation to the first layer of co-adsorbed molecules. Indeed, this is supposed to give most of the effects due to the solvent because of the relatively large adsorption energy per molecule $(0.39 \mathrm{eV}$ for acetonitrile and $0.68 \mathrm{eV}$ for water, both at full coverage) [26]. In our simulation we apply periodic boundary conditions simulating a periodic $\mathrm{TiO}_{2}$ slab with a large density of adsorbed LO dyes.

\section{METHODS}

The most widespread and accurate tool for the ab-initio study of ground state properties of molecular and extended systems is DFT. DFT fails, however, in the determination of single particle excitation energies for which more sophisticated techniques based on MBPT are needed.

The state of the art method for the evaluation of quasiparticle (QP) energy levels is the GW approach [27], in which QP energy levels are computed in a perturbative way starting from a DFT calculation. Here, we use the oneshot $\mathrm{G}_{0} \mathrm{~W}_{0}$ scheme in its simpler diagonal form employing the GWL package of the Quantum-Espresso (QE) suite of plane-waves pseudopotentials DFT codes. The $\mathrm{G}_{0} \mathrm{~W}_{0}$ scheme has been used by several authors [8,28-30] obtaining good results for different bulk $\mathrm{TiO}_{2}$ crystals. Similarly a $\mathrm{G}_{0} \mathrm{~W}_{0}$ study of crystalline dye sensitized $\mathrm{TiO}_{2}$ clusters supports the use of this approximation [6]. The GWL package uses a recently proposed numerical approach $[9,10]$ that allows us to overcome two main computational hindrances of this kind of calculation: sums over empty DFT orbitals and the size of the matrices involved in the scheme.

Our GW calculations were performed on top of DFT, on optimized structures obtained in Ref. [26]. We use periodically repeated simulation cells which are orthorhombic with edges of lengths: 19.3, 30.0, and 47.2 Bohr. This sets a vacuum layer between the top of the dye and the replica of the slab of $\sim 14$ Bohr. The total number of atoms is 156 in the case of the water solvent, and 174 in the case of the acetonitrile solvent, with a total number of electrons equal to 942 and 990 , respectively.

All DFT calculations were performed with the $p w . x$ code of the QE and using the Becke, Lee, Yang, and Parr (BLYP) exchange and correlation functional [31]. BLYP norm-conserving pseudopotentials were used and, for $\mathrm{Ti}$, the semicore $3 s$ and $3 p$ orbitals were treated as valence ones, a common practice in $\mathrm{GW}$ plane-waves pseudopotential calculations [32]. Therefore a high cutoff energy of $150 \mathrm{Ry}$ is required for the expansion of the Kohn-Sham orbitals in plane waves.

The parameters for the GW calculations were chosen to achieve an overall accuracy of $\sim 0.1 \mathrm{eV}$ in the determination of the QP energy levels for the states close to the HOMO and LUMO. In the case of the adsorbed L0 dye with the water solvent, we used an optimal basis set formed by the 6900 most important eigenvectors of an approximate polarizability operator at zero frequency. This corresponds (adopting the notation of Ref. [10]) to a cutoff energy $E^{*}=3 \mathrm{Ry}$ for the augmented plane waves and to an effective threshold 
$q=3.77 \mathrm{Bohr}^{3}$. In the case of the adsorbed L0 dye with the acetonitrile solvent, the polarizability basis was of 7700 elements, corresponding to $E^{*}=3 \mathrm{Ry}$ and $q=3.03 \mathrm{Bohr}^{3}$.

The frequency dependence of the response functions is fully taken into account by employing the analytic continuation technique [33]. We first obtain the expectation values of the self energy on imaginary frequency, and then we fit such functions with a three-pole expansion, obtaining the self-energy on the real frequency axis. We cross-checked the fitting procedure with calculations of the HOMO/LUMO levels done employing the contour deformation technique [34], recently implemented in the GWL package. It should be recalled that the GW QP energy levels for the HOMO and the LUMO correspond directly to the vertical ionization potential (IP) and to the vertical electron affinity (EA), respectively.

\section{RESULTS}

The most stable surface of anatase $\mathrm{TiO}_{2}$ and the one mainly exposed in photovoltaic applications is the (101) face $[35,36]$. We model it as a periodically repeated twolayers slab consisting of 96 atoms, as this was shown to be the minimal choice that could satisfactorily reproduce the electronic density of states of the bulk material [8]. We study a representative triphenylamine-based all-organic dye (LO) adsorbed on the anatase surface. We remark that the use of periodic boundary conditions implies the simulation of a periodic two-dimensional slab of $\mathrm{TiO}_{2}$ with a quite high surface density of adsorbed L0 molecules. Previous DFT calculations performed for a LO molecule adsorbed on an isolated $\left(\mathrm{TiO}_{2}\right)_{38}$ cluster exhibit similar geometries and energies [26]. Such a relative lack of dependence on the slab structure supports the validity of our results.

Either six molecules of water or acetonitrile are co-adsorbed on the surface, in order to study the effects of solvent and dye coadsorption on the alignment of electronic levels. In Ref. [26], the coadsorption of dye and water or acetonitrile $\left(\mathrm{CH}_{3} \mathrm{CN}\right)$ molecules was extensively studied at the DFT level, and it was found that, in both cases, the most stable geometry is a dissociated monodentate structure, with proton transfer to a nearby surface oxygen, as shown in Fig. 2. With acetonitrile the adsorption energy per $\mathrm{L} 0$ molecule is $0.70 \mathrm{eV}$ while the second most favorable binding mode is the dissociative bidentate
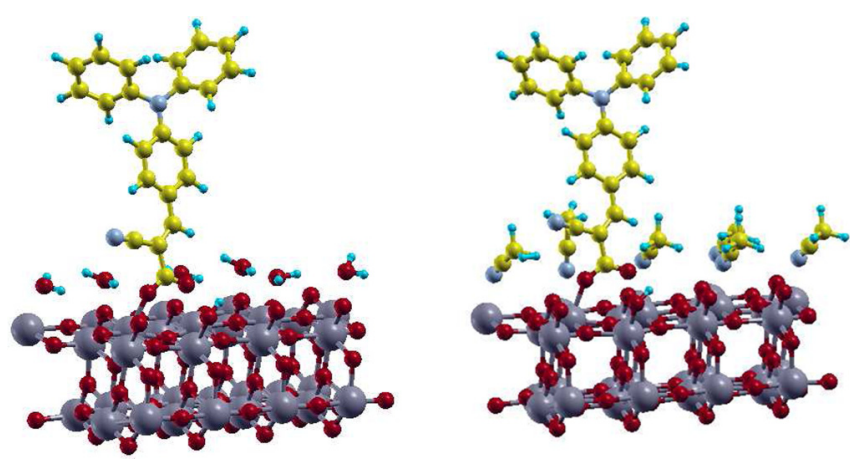

FIG. 2. (Color online) DSSC model structures: L0 dye adsorbed on the anatase $\mathrm{TiO}_{2}$ (101) surface in the presence of water (left) and acetonitrile (right).

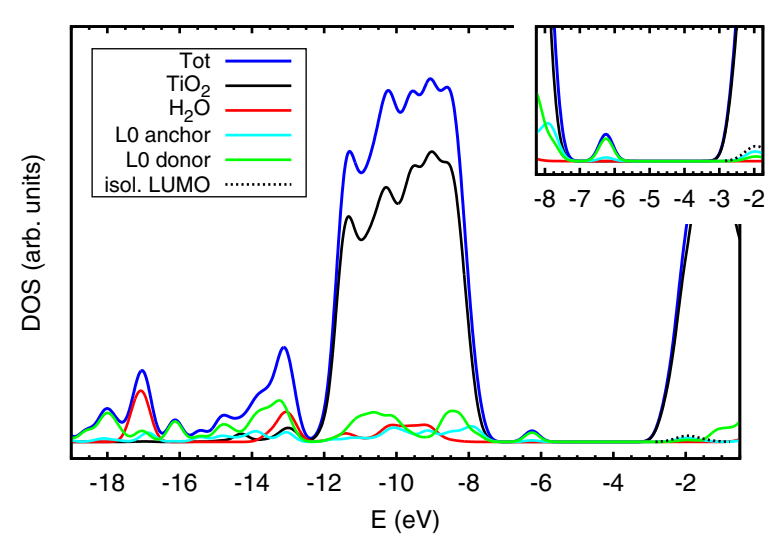

FIG. 3. (Color online) Calculated electronic DOS at the GW level for the $\mathrm{L} 0$ dye adsorbed on the anatase $\mathrm{TiO}_{2}$ (101) surface with water (blue) and projections on the atomic orbitals relative to: $\mathrm{TiO}_{2}$ (black), water (red), the donor group (green) and the anchor group (cyan) of the L0 dye. Black dotted line: projection on the isolated L0 dye LUMO. A Gaussian broadening of $0.25 \mathrm{eV}$ has been used and the energies are relative to the vacuum level. Inset: zoom to the energies close to the gap.

$(0.68 \mathrm{eV})$. In this investigation we consider the same model structures of Ref. [26].

\section{A. Electronic density of states}

In order to assess the effects induced by the presence of a solvent on the electronic properties, we analyze the electronic density of states (DOS) which can be obtained by broadening the calculated GW energy levels. In Fig. 3 we report the DOS for the adsorbed L0 dye with water, together with its projections on the atomic orbitals belonging to the slab, to the water molecules, and to the anchor and donor moieties of the dye.

The main feature between $\sim-12$ and $\sim-7 \mathrm{eV}$ is largely located on the $\mathrm{TiO}_{2}$ slab whereas, at lower energies, the DOS is due both to the dye and to the water molecules. The HOMO level of the system is at $-6.25 \mathrm{eV}$ with respect to the vacuum, and it is located on the dye, more precisely on the donor group. This is analogous to the cases of the adsorbed dye in vacuum and of an isolated L0 dye [8]. As in the case in vacuum, the HOMO level of the system (shown in Fig. 4) is very similar to the one in the gas phase: indeed, its squared projection onto the HOMO of the isolated LO molecule is equal to 0.980 . We point out that there is no single peak located mainly on the dye in the conduction part of the DOS close to the LUMO of the system.

We consider as $\mathrm{TiO}_{2}$ valence band maximum (VBM) the highest energy level of those occupied states which have a large slab character as we can see from the projections on atomic orbitals in Figs. 3 and 5. Analogously, we consider as $\mathrm{TiO}_{2}$ conduction band minimum (CBM) the lowest energy level of those unoccupied states which have a large slab character. We obtain the values of $-7.7 \mathrm{eV}$ and $-2.7 \mathrm{eV}$, for the VBM and the CBM, respectively. This corresponds to a substrate gap of $5.1 \mathrm{eV}$. This value is $0.8 \mathrm{eV}$ larger than the band gap of the clean two-layers slab [8], and it is close to the value $(4.9 \mathrm{eV})$ found in the case of the $\mathrm{LO}$ dye adsorbed on $\mathrm{TiO}_{2}$ in vacuum. 


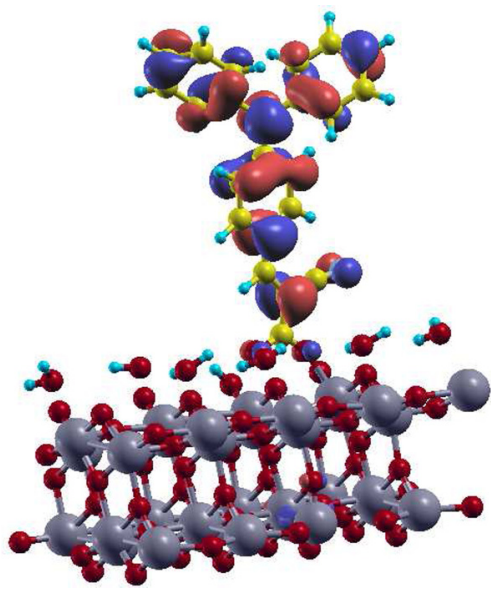

FIG. 4. (Color online) Isocharge surface relative to the HOMO of the $\mathrm{L} 0 @ \mathrm{TiO}_{2}+\mathrm{H}_{2} \mathrm{O}$ system.

This overestimation can be attributed to hybridization effects occurring because the size of the slab is comparable to that of the dye. It is worth noting that the band gap of the sole slab in vacuum $(4.3 \mathrm{eV})$ is $\sim 20 \%$ higher than the $\mathrm{GW}$ gap for bulk $\mathrm{TiO}_{2}$ anatase [8]. This should be ascribed to the limited size of the slab model $[30,37]$. Indeed, $G_{0} W_{0}$ calculated band gaps in bulk $\mathrm{TiO}_{2}$ anatase have been found in fair agreement with experiment with an overestimation of $\sim 5-10 \%[8,28-$ 30]. Moreover, studies focused on the dependence of the band gap of semiconductor clusters with respect to their diameter showed a remarkably slow convergence to the bulk value [38].

In Fig. 5 we display the electronic DOS for the adsorbed L0 dye with the acetonitrile solvent, together with the projections on the atomic orbitals of the slab, the acetonitrile molecules, and the L0 dye (for simplicity, we did not separate further the projection within the dye). We observe that the main features are analogous to the water case, and that the HOMO of the system is again localized on the dye, at an energy of $-6.4 \mathrm{eV}$.

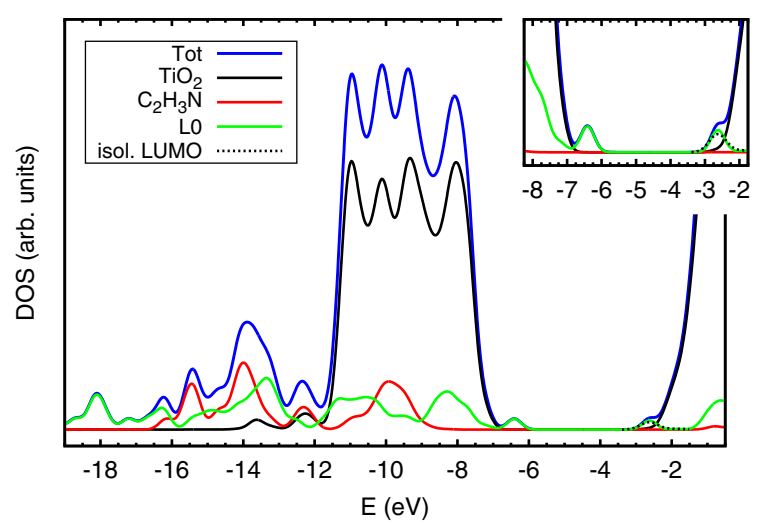

FIG. 5. (Color online) Calculated electronic DOS at the GW level for the $\mathrm{L} 0$ dye adsorbed on the anatase $\mathrm{TiO}_{2}$ (101) surface with acetonitrile (blue) and projections on the atomic orbitals relative to: $\mathrm{TiO}_{2}$ (black), acetonitrile (red) and the L0 dye (green). Black dotted line: projection on the isolated L0 dye LUMO. A Gaussian broadening of $0.25 \mathrm{eV}$ has been used and the energies are aligned to the vacuum. Inset: zoom to the energies close to the gap.
Interestingly, the VBM of the slab moves towards higher energies with respect to the case of water, being at $-7.2 \mathrm{eV}$, whereas the slab gap remains unchanged (5.1 eV). The "rigid" shift of the bands is a consequence of the electrostatic dipole due to the acetronitrile layer, similarly to what was found in Ref. [23] for the case of adsorbed molecules on CdSe.

It is worth noting that in the conduction part of the DOS there is one state mainly localized on the dye. This state corresponds to the LUMO+2 level in the DFT calculation, but after the GW correction it becomes the LUMO of the system at $-2.6 \mathrm{eV}$. This effect is due to the presence of the highly polar acetonitrile, and it does not occur with water.

\section{B. Energy-level alignment}

The energy levels of the dye and of the semiconductor should be properly aligned, as depicted in Fig. 1, in order to comply with the working principles of the cell. It is therefore important to assess our results in terms of the relative alignment of the energy levels of the different parts of the system.

In the case of the adsorbed dyes, we cannot find any empty states which could be clearly assigned to the molecular L0 LUMO, although there is dye-LUMO character in these states. We can rigorously obtain the LUMO energy of the adsorbed dye by calculating the projection of the DOS onto the LUMO of the isolated L0 molecule [8]. Indeed, in the isolated dye the first optical absorption peak has a HOMO-LUMO character, meaning that light effectively couples these two states. As the highest occupied state of our system has a high projection on the isolated molecule HOMO, in a first approximation we can assume that light couples this state with empty ones according to their projection on the isolated molecule LUMO.

Therefore, the (effective) LUMO energy of the adsorbed dye is estimated as the weighted average of the calculated QP energies $\epsilon_{i}^{\mathrm{QP}}$ :

$$
E_{\mathrm{LUMO}}=\frac{\sum_{i} \epsilon_{i}^{\mathrm{QP}} p_{i}}{\sum_{i} p_{i}}
$$

where $p_{i}$ is the squared projection of the $i$ th empty state of the adsorbed dye system over the LUMO of the isolated LO molecule. In Figs. 3 and 5 the distribution of $p_{i}$ as a function of energy is shown together with the DOS and PDOS, for the case of water and acetonitrile, respectively. This procedure yields a QP energy for the dye LUMO of $-1.88 \mathrm{eV}$ for the case of water and $-2.52 \mathrm{eV}$ for the case of acetonitrile. The latter is almost degenerate, within the precision of our GW calculations, with the LUMO of the entire solvent-dye-slab system which has a dye character as discussed in Sec. III A.

In the following, we summarize the positions, with respect to the vacuum level, of the HOMO and LUMO of the dye, and of the VBM and CBM of the $\mathrm{TiO}_{2}$. They are reported in Fig. 6, where the vacuum (from Ref. [8]), water, and acetonitrile cases are compared both for the DFT-BLYP and for the GW calculations.

As mentioned earlier, the semiconductor band gap for the clean slab is $\sim 20 \%$ larger than that of the bulk, and it becomes larger for the case of adsorbed molecules. However, we notice that: (a) the GW VBM of the clean slab is $-8.3 \mathrm{eV}$, close to the available experimental results for rutile $\mathrm{TiO}_{2}(-8.4 \mathrm{eV}$ from Ref. [39] and $-8.0 \mathrm{eV}$ from Ref. [40]), and (b) the VBM 

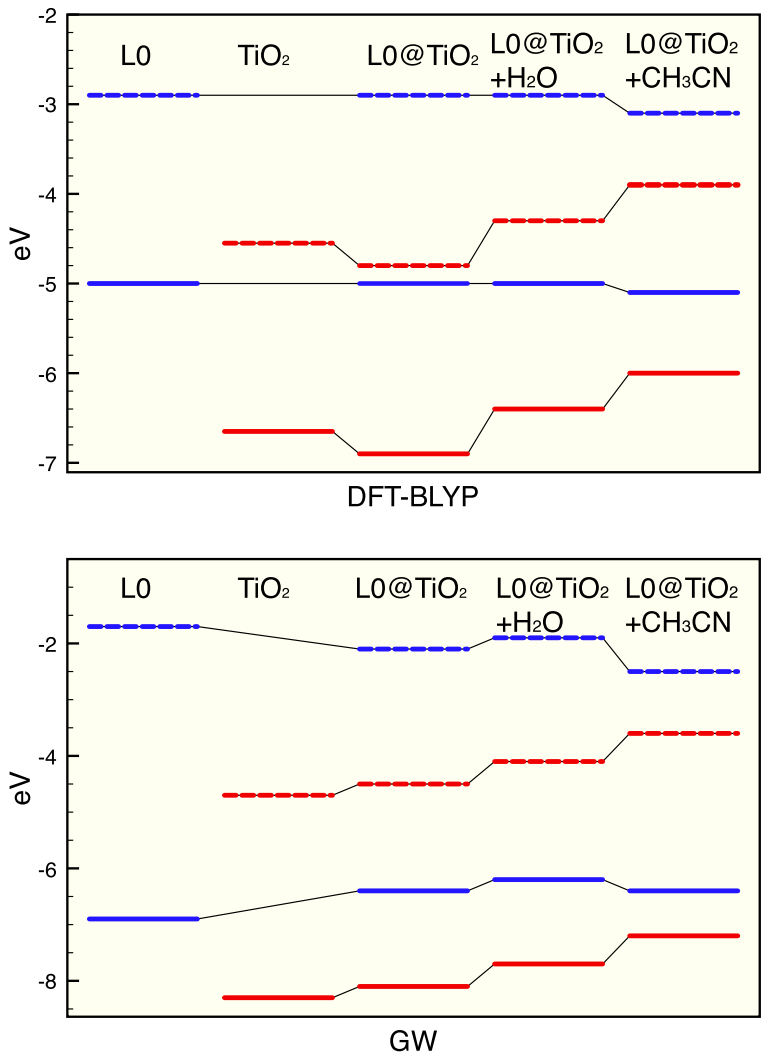

FIG. 6. (Color online) Energy levels with respect to the vacuum of the LO HOMO (blue bold) and LUMO (blue dashed) and VBM (red bold) and CBM (red dashed) of the $\mathrm{TiO}_{2}$ slab. The thin black line is a guide for the eye.

of the adsorbed system, without any solvent, is $-8.1 \mathrm{eV}$, close to the value found for the clean slab. We conclude that the VBM is fairly well described by our calculations. Therefore, we estimate the $\mathrm{CBM}$ of $\mathrm{TiO}_{2}$ in the adsorbed systems by taking the value of the VBM and adding the gap calculated for the bulk anatase $(-3.63 \mathrm{eV})$. A justification for this approach comes also from the fact that the DFT-BLYP gap of the bare and solvated $\mathrm{TiO}_{2}$ is found to vary less than $0.1 \mathrm{eV}$ (see Ref. [26] and references therein). The CBM values, reported in Fig. 6, were obtained with this procedure.

The upper panel of Fig. 6 shows that the DFT-BLYP HOMO-LUMO gap of the dye stays essentially the same after adsorption, in both the water and the acetonitrile cases. The dye HOMO and LUMO levels, in the presence of acetonitrile, have a small downward shift of $0.1 \mathrm{eV}$ and $0.2 \mathrm{eV}$ respectively, due to a dipole interaction with acetonitrile.

This picture changes significantly when many-body effects are included: The LO HOMO-LUMO gap, in the case of the water solvent, is reduced by about $0.9 \mathrm{eV}$ with respect to the gas phase, similarly to the case without solvent. This is ascribed to the interaction with the image charge, as it is found also for molecules physisorbed on metallic surfaces [41], but with a less pronounced effect because of the lower screening at a semiconductor surface. In the presence of the acetonitrile solvent, the GW-calculated LO HOMO-LUMO gap is reduced by a larger amount $(1.3 \mathrm{eV})$ : The HOMO moves up by about $0.4 \mathrm{eV}$, while the LUMO is pushed towards lower energies than in the water case. This is due to the presence of $\mathrm{CH}_{3} \mathrm{CN}$, which stabilizes the LUMO and polarizes the medium.

The analysis of the $\mathrm{TiO}_{2}$ levels reveals a strong effect due to the presence of the solvent. In fact, while the VBM of the adsorbed system in vacuum is essentially the same as in the isolated slab, we find an upward shift when water or acetonitrile are adsorbed on the surface. In particular, the CBM (calculated as described previously) value jumps from $-4.5 \mathrm{eV}$ (no solvent) to $-4.1 \mathrm{eV}$ (water solvent) or $-3.6 \mathrm{eV}$ (acetonitrile solvent). This is consistent with the upward shifts found, using DFT-BLYP, in Ref. [26], and it is related to the fact that both $\mathrm{H}_{2} \mathrm{O}$ and $\mathrm{CH}_{3} \mathrm{CN}$ have a donor character, leading to an increase in the negative charge on the $\mathrm{TiO}_{2}$ surface. Moreover, the dipole of the acetonitrile molecules points with the negative charge towards the $\mathrm{TiO}_{2}$ surface, therefore the upward shift is larger in this case. Such a trend going from water to acetonitrile is also found in experimental studies on $\mathrm{TiO}_{2}$ flat band potentials [42].

\section{Open-circuit voltage}

Qualitatively, GW confirms the DFT picture concerning the solvent effects on the alignment of the energy levels. However, the GW method allows us to quantitatively estimate the magnitude of such energy level shifts, and thus it is possible to estimate the maximum achievable cell $V_{\text {oc }}$.

Neglecting recombination effects, the maximum attainable open-circuit voltage $V_{\mathrm{oc}}^{\max }$ is given by the difference between the electron quasi-Fermi level of the semiconductor and the red-ox potential of the mediator, as shown in Fig. 1. It has been shown [43] that in colloidal $\mathrm{TiO}_{2}$ screened by an electrolyte band bending is negligible as internal electric fields are virtually absent. Hence, such effects are not considered in our modeling.

As the red-ox couple was not included in our simulation, we consider the standard red-ox potential $\left(E^{0}\right)$ of the $\mathrm{I}^{-} / \mathrm{I}_{3}^{-}$ couple measured in water [44] and in acetonitrile [45]. The red-ox potential is defined with respect to a reference electrode, usually the normal hydrogen electrode (NHE), which has a value of $-4.44 \mathrm{~V}$ with respect to the vacuum in water and $-4.43 \mathrm{~V}$ in acetonitrile [46].

The results are summarized in Table I. We obtain a value of $0.9 \mathrm{~V}$ for $V_{\mathrm{oc}}^{\max }$ in water, and a value of $1.1 \mathrm{~V}$ for the $V_{\mathrm{oc}}^{\max }$ in in acetonitrile. The experimental values for $\mathrm{TiO}_{2}$ DSSCs with the iodide/triiodide electrolyte in acetonitrile range from 0.65 to $0.9 \mathrm{~V}$ (see Refs. [45] and [47]), i.e., only a few tenths of volts below the predicted $V_{\mathrm{oc}}^{\max }$.

TABLE I. Maximum attainable open-circuit voltage of the cell $\left(V_{\mathrm{oc}}^{\max }\right)$ with water and acetonitrile solvent from $\mathrm{GW}$ calculations.

\begin{tabular}{lcc}
\hline \hline & $\mathrm{H}_{2} \mathrm{O}$ & $\mathrm{CH}_{3} \mathrm{CN}$ \\
\hline $\mathrm{CBM}(\mathrm{eV})$ & $-4.1^{\mathrm{a}}$ & $-3.6^{\mathrm{a}}$ \\
$E^{0}\left(\mathrm{I}^{-} / \mathrm{I}_{3}^{-}\right)(\mathrm{V})$ & $0.53^{\mathrm{b}}\left(-4.97^{\mathrm{a}}\right)$ & $0.31^{\mathrm{b}}\left(-4.74^{\mathrm{a}}\right)$ \\
$V_{\mathrm{oc}}^{\max }(\mathrm{V})$ & 0.9 & 1.1 \\
\hline \hline
\end{tabular}

${ }^{\mathrm{a}}$ Relative to vacuum.

${ }^{\mathrm{b}}$ Relative to NHE. 


\section{CONCLUSIONS}

We have shown how GW calculations, when applied to realistic, albeit simplified, models of DSSCs, are capable of describing overall the energy-level alignment required for the working principles of dye-sensitized solar cells. For the dye levels, in the case of water solvent we found results in substantial agreement with the ones previously found without the solvent, showing that the presence of a few water molecules on the semiconductor surface does not induce considerable modifications. In the case of the acetonitrile solvent, on the other hand, we see that the dye LUMO state is pushed towards lower energies at the GW level. This can be ascribed to the higher polarizability of the system and to the dipole interaction with the solvent molecules. As for the semiconductor levels, we found an upward shift in the CBM, more pronounced in acetonitrile than in water, in agreement with previous theoretical and experimental studies. This enhances the performance of the DSSC, through an increase in the open-circuit voltage $V_{\text {oc }}$.
The feasibility of accurate GW calculations on realistic models of dye-sensitized interfaces, including solvent molecules, will allow researchers in the field to estimate from first principles the main physical characteristics of these devices. Important will be the inclusion of the electron-hole coupling when evaluating the threshold for optical absorption. Within many-body perturbation theory, this can done with the Bethe-Salpeter equation scheme [48]. Another target for future work will be the inclusion in the simulations of an explicit solvent at room temperature. However, this would be considerably more computationally expensive because of the fourth-power scaling of the computational cost of GW schemes with respect to system size.

\section{ACKNOWLEDGMENTS}

The calculations have been performed at the CINECA HPC facility through the PRACE allocation Pra04 812. E.M. and F.D.A. thank FP7-ENERGY-2010, Project $2 \overline{62920}$ "ESCORT" for financial support.
[1] B. O’Regan and M. Grätzel, Nature (London) 352, 737 (1991).

[2] M. A. Green, K. Emery, Y. Hishikawa, and W. Warta, Prog. Photovoltaics 18, 144 (2010).

[3] J. Bisquert, Chem. Phys. Chem. 12, 1633 (2011).

[4] G. Onida, L. Reining, and A. Rubio, Rev. Mod. Phys. 74, 601 (2002).

[5] M. S. Hybertsen and S. G. Louie, Phys. Rev. B 34, 5390 (1986).

[6] N. Marom, J. E. Moussa, X. Ren, A. Tkatchenko, and J. R. Chelikowsky, Phys. Rev. B 84, 245115 (2011).

[7] C. E. Patrick and F. Giustino, Phys. Rev. Lett. 109, 116801 (2012).

[8] P. Umari, L. Giacomazzi, F. De Angelis, M. Pastore, and S. Baroni, J. Chem. Phys. 139, 014709 (2013).

[9] P. Umari, G. Stenuit, and S. Baroni, Phys. Rev. B 79, 201104(R) (2009).

[10] P. Umari, G. Stenuit, and S. Baroni, Phys. Rev. B 81, 115104 (2010).

[11] D. P. Hagberg, J.-H. Yum, H. Lee, F. De Angelis, T. Marinado, K. M. Karlsson, R. Humphry-Baker, L. Sun, A. Hagfeldt, M. Grätzel, and Md. K. Nazeeruddin, J. Am. Chem. Soc. 130, 6259 (2008).

[12] E. M. J. Johansson, T. Edvinsson, M. Odelius, D. P. Hagberg, L. Sun, A. Hagfeldt, H. Siegbahn, and H. Rensmo, J. Phys. Chem C 111, 8580 (2007).

[13] M. Hahlin, M. Odelius, M. Magnuson, E. M. J. Johansson, S. Plogmaker, D. P. Hagberg, L. Sun, and H. Rensmo, Phys. Chem. Chem. Phys. 13, 3534 (2011).

[14] D. P. Hagberg, T. Marinado, K. M. Karlsson, K. Nonomjura, P. Qin, G. Boschloo, T. Brink, A. Hagfeldt, and L. Sun, J. Org. Chem. 72, 9550 (2007).

[15] T. Marinado, K. Nonomura, J. Nissfolk, M. K. Karlsson, D. P. Hagberg, L. Sun, S. Mori, and A. Hagfeldt, Langmuir 26, 2592 (2010).

[16] T. Marinado, D. P. Hagberg, M. Hedlund, T. Edvinsson, E. M. J. Johansson, G. Boschloo, H. Rensmo, T. Brinck, L. Sun, and A. Hagfeldt, Phys. Chem. Chem. Phys. 11, 133 (2009).
[17] C. J. Barbé, Arendse, P. Comte, M. Jirousek, F. Lenzmann, V. Shklover, and M. Grätzel, J. Am. Ceram. Soc. 80, 3157 (1997).

[18] S. Meng, J. Ren, and E. Kaxiras, Nano Lett. 8, 3266 (2008).

[19] M. Ni, M. K. H. Leung, D. Y. C. Leung, and K. Sumathy, Sol. Energy Mater. Sol. Cells 90, 2000 (2006).

[20] F. Schiffmann, J. Hütter, and J. Vande Vondele, J. Phys. Condens. Matter 20, 064206 (2008).

[21] F. De Angelis, S. Fantacci, and R. Gebauer, J. Phys. Chem. Lett. 2, 813 (2011).

[22] S. A. Kislenko, R. H. Amirov, and I. S. Samoylov, J. Phys. Chem. C 117, 10589 (2013).

[23] S. Yang, D. Prendergast, and J. B. Neaton, Nano Lett. 12, 383 (2012).

[24] K. Onda, B. Li, and H. Petek, Phys. Rev. B 70, 045415 (2004).

[25] A. F. Bartelt, R. Schütz, C. Strothkämper, I. Kastl, S. Janzen, D. Friedrich, W. Calvet, G. Fuhrmann, D. Danner, L-P. Scheller, G. Nelles, and R. Eichberger, Appl. Phys. Lett. 104, 223902 (2014).

[26] E. Mosconi, A. Selloni, and F. De Angelis, J. Phys. Chem. C 116, 5932 (2012).

[27] L. Hedin, Phys. Rev. 139, A796 (1965).

[28] L. Chiodo, J. M. García-Lastra, A. Iacomino, S. Ossicini, J. Zhao, H. Petek, and A. Rubio, Phys. Rev. B 82, 045207 (2010).

[29] M. Landmann, E. Rauls, and W. G. Schmidt, J. Phys. Condens. Matter 24, 195503 (2012).

[30] Wei Kang and Mark S. Hybertsen, Phys. Rev. B 82, 085203 (2010).

[31] A. D. Becke, Phys. Rev. A 38, 3098 (1988); C. Lee, W. Yang, and R. G. Parr, Phys. Rev. B 37, 785 (1988).

[32] P. Umari and S. Fabris, J. Chem. Phys. 136, 174310 (2012).

[33] M. M. Rieger, L. Steinbek, I. D. White, H. N. Rojas, and R. W. Godby, Comput. Phys. Commun. 117, 211 (1999)

[34] A. Fleszar and W. Hanke, Phys. Rev. B 56, 10228 (1997). 
[35] M. Pastore and F. De Angelis, Phys. Chem. Chem. Phys. 14, 920 (2012).

[36] C. Anselmi, E. Mosconi, M. Pastore, E. Ronca, and F. De Angelis, Phys. Chem. Chem. Phys. 14, 15963 (2012).

[37] C. Freysoldt, P. Eggert, P. Rinke, A. Schindlmayr, and M. Scheffler, Phys. Rev. B 77, 235428 (2008).

[38] A. J. Williamson, J. C. Grossman, R. Q. Hood, A. Puzder, and G. Galli, Phys. Rev. Lett. 89, 196803 (2002).

[39] K. D. Schierbaum, S. Fischer, M. C. Torquemada, J. L. de Segovia, E. Román, and J. A. Martín-Gago, Surf. Sci. 345, 261 (1996).

[40] G. Xiong, R. Shao, T. Droubay, A. Joly, K. Beck, S. Chambers, and W. Hess, Adv. Funct. Mater. 17, 2133 (2007).
[41] J. B. Neaton, M. S. Hybertsen, and S. G. Louie, Phys. Rev. Lett. 97, 216405 (2006)

[42] G. Redmond and D. Fitzmaurice, J. Phys. Chem. 97, 1426 (1993).

[43] A. Hagfeldt and Michael Grätzel, Chem. Rev. 95, 49 (1995).

[44] P. Atkins, Physical Chemistry, 6th ed. (W. H. Freeman and Company, New York, 1997).

[45] T. Le Bahers, F. Labat, T. Pauporté, and I. Ciofini, Phys. Chem. Chem. Phys. 12, 14710 (2010).

[46] A. A. Isse and A. Gennaro, J. Phys. Chem. B 114, 7894 (2010).

[47] M. Grätzel, Acc. Chem. Res. 42, 1788 (2009).

[48] M. Rohlfing and S. G. Louie, Phys. Rev. B 62, 4927 (2000). 